myositis, vasculitis, and gout. We herein present a patient mimicking SLE who was diagnosed with HIV.

Methods A 40-year-old man presented with a 3-month history of oral lesions and a 2-month history of blurry vision, vertigo, speech difficulty, and ataxic gait. His past medical history was unremarkable. His vital findings were within normal ranges. There were two oral lesions that were greater than $10 \mathrm{~mm}$ in diameter localized on the hard palate and tongue. Cerebellar tests including gait, finger-to-nose, and heel-to-shin were abnormal. He could not walk in tandem gait. However, muscle strength and sensory exam were normal.

On laboratory outcomes, fasting blood glucose, blood urea nitrogen, creatinine, and thyroid-function tests were normal. The rest of laboratory data were as follows: ALT $129 \mathrm{U} / \mathrm{L}$, AST $102 \mathrm{U} / \mathrm{L}$, GGT $72 \mathrm{U} / \mathrm{L}$, hemoglobin $12.3 \mathrm{mg} / \mathrm{L}$, WBC $3.6 \times 10^{3} / \mathrm{mcL}$, neutrophil count $1.8 \times 10^{3} / \mathrm{mcL}$, lymphocyte count $1.2 \times 10^{3} / \mathrm{mcL}$, CRP $1.5 \mathrm{mg} / \mathrm{dl}$ (normal, $\leq 5 \mathrm{mg} / \mathrm{dl}$ ), ESR $42 \mathrm{~mm} / \mathrm{h}$.

Cranial MRI was reported as multiple hyperintense spots located on supratentorial white matter, corpus callosum, and brainstem on standard T2-weighted MRI sequence. On neurological consultation, these lesions were interpreted as encephalitis. Additionally, he was consulted to Ophthalmologist due to blurry vision. No pathological finding was found. He was also for autoimmune disorders causing cerebral involvement. Thus, ANA, ENA, anti ds DNA, ANCA were investigated. All autoantibodies, hepatitis screening, CMV, EBV, parvovirus B 19 were found negative. On the other hand, HIV positivity was detected by ELISA. Consequently, the patient was transferred to department of Infectious diseases.

Conclusion HIV causes autoimmune and systemic disorders via triggering immune dysregulation. The frequency of these autoimmune diseases has ranged from $1 \%$ to $60 \%$ according to literature. Both HIV and the treatment of HIV can cause rheumatologic findings.

\section{P47 COGNITIVE IMPAIRMENT IN JSLE - THE ROLE OF INFLAMMATION}

${ }^{1}$ Beatriz Silva, ${ }^{2}$ Sara Ganhão, ${ }^{2}$ Mariana Rodrigues, ${ }^{2}$ Francisca Aguiar, ${ }^{2}$ Iva Brito, ${ }^{1}$ Margarida Figueiredo-Braga. 'Dept. of Clinical Neurosciences and Mental Health, Faculty of Medicine, University of Porto, Porto; ${ }^{2}$ Young Adult and Pediatric Rheumatology Unit, Centro Hospitalar e Universitário do Hospital de São João, Porto, Portugal

10.1136/lupus-2020-eurolupus.95

Background Patients with juvenile-onset Systemic Lupus Erythematosus (jSLE) cope with physical and neuropsychiatric symptoms which may interfere with social and professional activities. Mild cognitive impairment may negatively impact quality of life and academic performance. We intend to assess cognitive state in JSLE and to explore laboratory and clinical markers associated with the presence of mild cognitive impairment.

Methods Thirty jSLE patients, currently aged $\geq 16$ years, followed in an outpatient's unit performed the Mini Mental State Examination (MMSE) for cognitive testing; clinical and laboratory measures were collected from clinical records. Juvenile-onset was defined as age at diagnosis $<18$ years. Statistical analyses were performed with SPSS software, version 25 .
Results The mean age was 22.8(5.3) years with 90\% females. Patients had a mean of $12.7(2.3)$ years of formal education. A mean MMSE of 27.7 (1.9) was found, and 23.3\% of the population showed mild cognitive impairment. MMSE scores were negatively correlated with C-Reactive Protein (CRP) $(\mathrm{r}=-0.38, \mathrm{p}=0.44)$ and platelet count $(\mathrm{r}=-0.37, \mathrm{p}=0.44)$. Additionally, patients presenting positive anti-SSA $(n=10)$, anti-RNP $(n=4)$ and anti-SM $(n=4)$ autoantibodies, scored significantly lower MMSE scores compared to patients without these autoantibodies $(\mathrm{p}=0.029 ; \mathrm{p}=0.017$ and $\mathrm{p}=0.017$, respectively).

Conclusions Our population showed a low mean MMSE score, regardless of their educational level and age. The consistent relationship between the presence of cognitive impairment, higher inflammatory activity and autoantibodies frequently associated with neuropsychiatric involvement in jSLE seems to point to the need to screen jSLE populations for mild cognitive impairment with larger studies being required.

\section{P48 ARE NEW 2019 SLE EULAR/ACR CLASSIFICATION CRITERIA USEFUL IN PATIENTS WITH RHUPUS?}

Beatriz Frade-Sosa, Javier Narváez, Tarek Salman-Monte, Vera Ortiz-Santamaria, Vicente Torrente-Segarra, Ivan Castellvi, Berta Magallares, Raul Castellanos-Moreira, Delia Reina, Sonia Minguez, Meritxell Sallés, Maria García Manrique de la Lara, Sonia Ordoñez, Elena Riera, Jose A Gómez-Puerta, on behalf of the CAPITCAT-MAS Study Group, Catalonia, Spain

\subsection{6/lupus-2020-eurolupus.96}

Background Concomitant presence of systemic lupus erythematous (SLE) and rheumatoid arthritis (RA) in the same patient is known as Rhupus. Poliautoimmunity is not an uncommon phenomenon reported, but only a small series of patients have been described so far with Rhupus. This study aimed to explore the utility of 2019 EULAR/ACR criteria in patients with Rhupus.

Methods Cross-sectional study conducted in 11 Rheumatology Departments at Catalonia (Spain). We included prevalent cases classified as Rhupus (RA ACR/EULAR 2010 and SLE-ACR 1997). Data of patients with SLE were selected as control in a ratio $2: 1$, matched by sex.

Results 120 patients were included, 40 Rhupus and 80 SLE alone as controls. 95\% were female, with a mean age of 51 yrs and mean of 13 yrs. The 2019 SLE EULAR/ACR criteria were met in $92,5 \%$ of the Rhupus and in the $96,3 \%$ of the SLE cohort $(p>0,05)$. Numerically, criteria score was higher in those patients with 'pure' SLE than Rhupus (21.3 \pm 7.0 vs $18.8 \pm 6.5, \mathrm{p}=\mathrm{NS}$ ). Excluding articular domain, there were no significant differences among those who meet new criteria $(65 \%$ Rhupus vs. $77 \%$ SLE $(p>0.05))$. Proteinuria was the only domain significantly more common in patients with SLE alone. No other differences were found in other clinical or serological characteristics.

Conclusion More than $90 \%$ of patients classified as Rhupus criteria fulfilled new SLE criteria. This study shows that the new 2019 SLE EULAR/ACR criteria are not useful to differentiate SLE and Rhupus patients. Further investigations are needed to measure their utility in clinical practice for detecting overlap presentation of RA and SLE. 